\title{
Guidelines for basic techniques in vascular access intervention therapy（VAIVT）
}

\section{Authors}

Matsuura Katsuhiko: Department of Radiology, Saitama Medical Center, Jichi Medical University, kmatsur@omiya.jichi.ac.jp Gotoh Yasuo: Shunt Clinic Sendai Higashi, MFX03261@ nifty.ne.jp

Sadaoka Shunichi: Department of Radiology, Jikei University Kashiwa Hospital, sada_s@jikei.ac.jp Takase Kei: Department of Diagnostic Radiology, Tohoku University, ktakase@ rad.med.tohoku.ac.jp

Narimatsu Yoshiaki: Department of Radiology, Kawasaki Municipal Hospital, nari@mfpm.jp 


\begin{abstract}
Vascular access intervention therapy (VAIVT) is an interventional therapy that is an essential part of dialysis as it allows for long-term continuous vascular access (VA). This Guideline is intended to serve as an aid for solving of actual technical problems encountered when performing VAIVT. After reviewing the basics of VAIVT, we listed the various VAIVT techniques in clinical question (CQ) format and provided descriptions. Specifically, these descriptions contain the indications, contraindications, VA tests, methods of using VAIVT devices, complications associated with VAIVT, and most of the issues related to VAIVT measures and practices. We also ensured that contents were consistent with the Guideline of Vascular Access Construction and Repair for Chronic Hemodialysis produced by the Japanese Society for Dialysis Therapy. We hope that this Guideline is useful to medical practitioners involved in VAIVT and contribute to maintaining vascular access for dialysis patients.
\end{abstract}

\title{
Key Words
}

Hemodialysis, Vascular Access, Vascular Access Intervention Therapy (VAIVT)

\section{Introduction}

Access to the bloodstream that is utilized for blood removal and replacement during dialysis is known as "vascular access," a nomenclature that is recognized worldwide. However, vascular access, particularly in the case of endovascular therapy performed using an arteriovenous fistula (AVF) or an arteriovenous graft (AVG), is also known by the following terms, indicating that a single common term does not exist: percutaneous dialysis interventions; endovascular repair; angioplasty for hemodialysis access; endovascular intervention for the vascular access; percutaneous intervention; percutaneous treatment of hemodialysis fistulas and grafts; interventional radiology of vascular accesses; and others. In 2007, the Japanese Society of Dialysis Therapy recommended that interventional therapies and endovascular therapies utilizing vascular access be commonly referred to as "vascular access intervention therapy" (VAIVT), and as a result, this term has come into common use in Japan. ${ }^{1}$ Thus, the term "VAIVT" is utilized in this Guideline.

This Guideline is intended to serve as an aid in solving actual technical problems that arise when performing VAIVT. For information regarding the basics of vascular access, please refer to the Guideline of Vascular Access Construction and Repair for Chronic Hemodialysis, published by the Japanese Society for Dialysis Therapy (44: 856-937). ${ }^{2}$ Of particular interest to VAIVT are (1) stenosis and occlusion, (2) aneurysms, and (3) venous hypertension, which are listed in Chapter 5 Managing Trouble Related to Vascular Access (pp. 906-913)

This Guideline is created by five experts who regularly perform VAIVT and who were members of the Japanese Society for Interventional Radiology as of 2016. These five experts formed a working group that created the basic therapeutic contents based on consensus. This basic manuscript was subsequently revised after a search of the relevant medical literature. The Japanese Society for Dialysis Therapy Guideline Committee granted its consent to the use of these Guidelines as a supplement to their own Guideline.

The references utilized in this Guideline originally included 500 papers published between 2000 and 2010 that were extracted from PubMed in January 2011 using the search term (keyword) "hemodialysis." Of these 500 papers, 93 that were related to VAIVT techniques were selected. We plan to revise these Guidelines every few years by repeating searches of relevant medical literature.

\section{The current state of hemodialysis in Japan}

Vascular access (VA) utilized in hemodialysis was previously known as "dialysis shunt." With the widespread use of vascular access intervention therapy (VAIVT), the term "access" came into common use. ${ }^{3,4,5,6}$ However, dialysis medical staff were in the habit of referring to an arteriovenous fistula, which utilizes the patient's own blood vessels, as a "shunt" and an arteriovenous graft, which utilizes an artificial tube, as a "graft." The listing for the number of health insurance points assigned for VAIVT and related surgery still uses the term "shunt".

As of the end of December 2015, there were more than 320,000 (324,986 to be exact) patients on continuous hemodialysis in Japan. The most common primary disease was diabetic nephropathy (38.1\%), the mean age of the patients was 67.5 years, and the mean age at the time dialysis therapy was begun was 69.0 years, indicating that the mean age of dialysis patients is increasing. ${ }^{7}$ Investigation of the types of VA being utilized indicated that $89.7 \%$ were using AVF and $7.1 \%$ were using AVG, demonstrating that AVF is the dominant procedure in Japan.

AVF involves the creation of an artificial anastomosis between a native artery and vein. As a vein is used as an artery through the redirection of arterial blood flow into a surface vein and a dialysis needle has to be inserted three times per week to perform hemodialysis, AVF has VA-related problems such as stenosis due to intimal hyperplasia cicatricial stricture. Naturally, VA is a lifeline for dialysis patients. Thus, VAIVT was an essential interventional therapy in the field of dialysis medicine that allows the maintenance of long-term VA functionality. ${ }^{3,4,5,6,8}$ 


\section{Types of problems associated with VA (Figs. 1 and 2)}

Stenosis can occur upstream (due to the fact that an anastomosis is artificially created between an artery and vein, blood flow on the anastomosis side is considered "upstream" and blood flow on the heart side is considered "downstream") or downstream. Instances of stenosis most commonly occur near the anastomosis on the upstream side (juxta-anastomotic site). Downstream stenosis is usually medial of the puncture site, and therefore since there is little to no effect on blood removal, discovery of this type of stenosis is often delayed.

Occlusion includes both thrombotic occlusion and non-thrombotic occlusion. The most common cause of thrombotic formations is stenosis, although dehydration, hypotension, and hypercoagulability are contributing factors. Non-thrombotic occlusion occurs when a long-term stenosis progresses to an advanced degree and the VA vein becomes occluded without accompanying thrombosis. Since this condition can become chronic, in many cases collateral routes are formed. VA occlusion is an extension of VA stenosis. Appropriate treatment of VA stenosis can prevent VA occlusion.

Cases at risk of VA failure include those with poor blood removal, puncturing difficulty, shifting of the puncture site upstream, delayed hemostasis, vessel pain, elevated venous pressure during dialysis, and decreased dialysis efficacy. Prior to performing VAIVT, a check to determine if the patient is at risk of VA failure and a check of the previous three times of dialysis records. Dialysis records are used to check symptoms such as variations in blood pressure, blood flow rate (QB), venous pressure, puncture site, and pain experienced during dialysis.

\section{VA examination}

Inspection includes a check of skin color from the upper arms to the hands and fingers, a check for edema, and a check of venous and collateral blood flow routes. Palpation includes a check of brachial artery and radial artery pulse followed by examination of the course, diameter, and tension of the vein starting at the anastomosis site and advancing downstream. Venous distension under avascularization is also checked.

Auscultation consists of checking for high-pitch sound. The maturation of VA may be unsatisfactory even in cases in which blood flow is audible. The blood flow will give off an intermittent strong trilling sound on the immediately cardiac side of the stenosis site In many cases, this sound decreases drastically as blood continues along the cardiac side.

Thrombotic occlusion can be hardly palpated. Non-thrombotic occlusion is identified by the interruption of the pulse. Collateral routes can be identified from the site of pulse interruption. At times, a non-thrombotic occlusion site feels like it has a funicular structure. Ultrasound is an important tool used for increasing precision during follow-up examinations.

\section{Diagnostic images required to assess VA function}

\section{1) Ultrasound ${ }^{9}$}

Ultrasound is the basic examination that is required to conduct evaluation prior to VAIVT and periodic follow-up examinations of the VA. In cases of VA, a 7 to $10 \mathrm{MHz}$ high-frequency linear probe is used on surface veins. In cases of AVF, examination is conducted from the brachial artery to the arteriovenous anastomosis and then the forearm and brachial veins. Blood vessel courses are examined using B-mode and color Doppler, and the features of the lesion are identified. Blood vessels are examined along both the long and short axes, and the vessel diameter, degree of stenosis, condition of the intima, calcification, and embolisms (hyperechoic/luminescent in comparison to the blood) are identified. Since the site of arteriovenous anastomosis presents a sharp angle, examination from multiple angles is required. If the diameter of the anastomosis is $2 \mathrm{~mm}$ or larger, the blood flow is preserved.

Occlusive lesions are assessed by deficiencies in the color signal and identifying the presence of emboli. Non-thrombotic occlusions are assessed by identifying occluded funicular blood vessel structures and collateral routes.

Measurement of flow volume (FV) and the resistance index (RI) are the basic methods used to assess functionality. Abnormality is indicated when the $\mathrm{FV}$ is at $500 \mathrm{ml} / \mathrm{min}$ or lower and the $\mathrm{RI}$ is at 0.6 or above. Once the $\mathrm{FV}$ reaches $350 \mathrm{ml} / \mathrm{min}$ or below, it becomes particularly difficult to maintain a QB of $200 \mathrm{ml} / \mathrm{min}$.

\section{2) VA angiography}

Angiography is performed using digital subtraction angiography (DSA). Although angiography is used in most cases of VAIVT, diagnostic angiography is required in cases in which VA assessment is complex and in cases in which central venous lesions are difficult to evaluate by ultrasound. Angiography consists of 1) Antegrade imaging from the brachial artery and 2) Retrograde imaging from the vein (avascularization is performed using blood pressure cuff to raise the systolic blood pressure at least 20 to $50 \mathrm{mmHg}$ ). In the case of retrograde imaging, avascularization is released after sufficient imaging from the anastomosis site to the artery and return of blood flow to the downstream vein is evaluated. In order to avoid arteriovenous overlap during contrast imaging of the forearm, images are made from at least two directions. The contrast medium used has an iodine concentration of $150 \mathrm{mgI} / \mathrm{ml}$. Arteriography uses 3 to $5 \mathrm{ml}$ and retrograde venography uses $10 \mathrm{ml}$.

In cases in which the use of iodine contrast medium is contraindicated, the same VA angiography can be performed using 20 to $50 \mathrm{ml}$ of negative contrast medium CO2 gas alone. ${ }^{4}$ However, care is required regarding the following points: 1) In cases 
in which there is retrograde flow through the anastomosis site from the brachial artery to the subclavian artery and then to the brachiocephalic artery, there is a danger that a gas embolism may cause a transient ischemic attack (TIA); 2) Total dose must be $200 \mathrm{ml}$ or less; and 3) A 3-minute interval is required between CO2 absorption and contrast imaging.

\section{Explanation of terms used in this guideline}

\section{1) VAIVT: Vascular Access Interventional Therapy}

Indicates all percutaneous therapies designed to manage problems related to vascular access for dialysis, including problems such as stenosis and occlusion.

\section{2) AVF: Arteriovenous fistula}

An anastomosis is surgically created between the patient's native artery and vein to ensure vascular access for dialysis. Normally, the anastomosis is created on the thumb side of the wrist between the radial artery and cephalic vein. However, it can also be created on the opposite side of the wrist between the ulnar artery and basilic vein, at the elbow between the brachial artery and cephalic vein, or at the anatomical snuffbox at the base of the thumb between the periphery of the radial artery and the periphery of the cephalic vein. In addition, AVF consisting of a V-V bypass using an artificial tube between a vein in the upper part of the forearm and a thick vein in the upper arm has recently come into use to remedy poor venous outflow due to non-thrombotic occlusion at the elbow, where AVF is normally created.

\section{3) AVG: Arteriovenous graft}

AVG is a type of vascular access for use in dialysis that is created by linking the patient's own artery and a vein using an implanted vessel. In many cases, the brachial artery at the elbow and either the basilica vein or a brachial vein in the inferior region of the upper arm are looped and connected using a graft that is implanted in the forearm. The graft is mainly made of either expanded polytetrafluoroethylene (ePTFE) or polyurethane. In some cases the patient's own vein (e.g. great saphenous vein) is used. A straight implanted graft is utilized to connect the radial artery at the wrist and one of the patient's own veins at either the elbow or inferior region of the upper arm. Implantation sites include looped artificial tubes in the forearm, the upper arm, and the thigh.

\section{4) Vascular access routes}

The blood flow that passes through the arteriovenous anastomosis in AVF and through the graft implanted between the artery and vein in AVG flows along the shortest route from the heart to re-entry into the heart. In AVF, the artery is defined as the portion up to the arteriovenous anastomosis and in AVG, the portion of the artery up to the transplanted graft is defined as the inflow artery. In AVF, the outflow vein is defined as the portion from the arteriovenous anastomosis to the heart. In AVG, the portion of the vein from the arteriovenous anastomosis of the implanted graft to the heart is defined as the outflow vein.

\section{5) Thrombotic occlusion}

This indicates a condition in which a thrombus develops in some part of the vascular access route and interrupts the blood flow in that region of the route. In general, in the case of AVF, this condition commonly occurs in the vein in the juxta-anastomotic site. However, in some cases, it may occur at a distant site from the anastomosis site due to the existence of stenosis or a blood vessel that serves as a collateral route. In the case of AVG, this condition commonly occurs in a region that extends from the entire length of the implanted graft to a region along the patient's own vein.

\section{6) Non-thrombotic occlusion}

This indicates a condition in which a stenosis develops in some part of the vascular access route, and as this stenosis enlarges the blood vessel lumen completely collapses, resulting in the interruption of blood flow in that region. In many cases, the presence of a collateral route allows upstream blood flow to continue, but thrombotic occlusion also sometimes occurs upstream (or downstream) of a non-thrombotic occlusion.

\section{7) Anatomical classification and blood flow direction of target vessels}

The region from the wrist to the elbow is defined as the forearm, the region from the elbow to a region peripheral to the confluence of the subclavian vein and the cephalic vein is defined as the upper arm, and the region from the confluence of the subclavian vein and the cephalic vein to the confluence of the brachiocephalic vein and the superior vena cava is defined as the central vein. For each of these regions, upstream and downstream blood flow directions are identified.

\section{[Clinical Questions (CQs) Regarding the Practice of VAIVT]}

As there were very few references that contained evidence based on randomized controlled trials (RCTs), we relied mainly on expert opinion. Determination of the specific contents of the CQs and the recommendation levels were made via the infor- 
mal consensus method of five committee members. The results were assessed externally by two experts not associated with this committee. In addition, we ensured conformity with the contents of the Guideline of Vascular Access Construction and Repair for Chronic Hemodialysis (The Japanese Society for Dialysis Therapy 44: 856-937).

\section{Recommendation level}

1: Strongly recommended

2: Weakly recommended

\section{Evidence level}
A: Strong
High degree of confidence in the anticipated efficacy
B: Moderate
Moderate degree of confidence in the anticipated efficacy
C: Weak
Limited degree of confidence in the anticipated efficacy
D: Very weak
Almost no confidence in the anticipated efficacy

\section{Clinical Questions (CQs) Specific to VAIVT Techniques - 15 Questions}

1. What are the indications for VAIVT?

2. What are the contraindications for VAIVT?

3. What methods are used to evaluate vascular access?

4. What are the arterial approach and venous approach? For what purpose is each used?

5 . How is a balloon catheter selected?

6. How is the balloon diameter selected?

7. How are percutaneous transluminal angioplasty (PTA) balloons dilated?

8. What is the endpoint for balloon dilation procedure?

9. How is blood vessel damage caused by the PTA balloon managed?

10. How is thrombotic occlusion treated?

11. What is the endpoint for thrombectomy?

12. What are the indications for stents?

13. How are stents selected?

14. How are stents placed?

15. What complications are associated with stent placement?

CQ1. What are the indications for vascular access interventional therapy (VAIVT)?

Cases in which the entire length of the artery, which is the inflow route for the vascular access, to the arteriovenous anastomosis (in AVG, the arterial anastomosis) and the central vein that is the downstream outflow route have stenosis or non-thrombotic occlusion that causes decreased blood flow, thrombotic occlusion, venous hypertension, or poor initial development after VA construction.

\section{Recommendation level: 1B}

\section{Description}

\section{Subjects and indications}

The subjects are lesions anywhere along the entire VA route - i.e. the entire length of the artery from the origin from the aorta to the arteriovenous anastomosis, the arteriovenous anastomosis (in the case of AVG, the entire graft), and the central vein (draining vein)up to the confluence with the vena cava. ${ }^{13,18,20,21,30,38,49,78,93}$ The types of lesions considered to be targets are stenosis, lesions requiring treatment, including both thrombotic and non-thrombotic occlusions. However, in cases involving a central artery or vein, which are likely to require emergency hemostasis if the blood vessel is damaged, each medical facility must determine whether VAIVT is indicated based on the facility's abilities, including whether they have covered stents for hemostasis or surgical backup available.

\section{Other indications}

VAIVT is indicated in the presence of symptoms other than those included in these Guidelines such as varices and steal syndrome. For example, in cases of subclavian steal syndrome caused by the stenosis of varicose vein and radial artery of the forearm, VAIVT can be indicated to relieve symptoms. There are also some cases in which a varix that has satisfactory outflow will shrink or disappear as a result of placement of a stent that straddles the varix.

In cases in which blood removal is difficult, determining whether removal of $200 \mathrm{ml} / \mathrm{min}$ of blood from the normal puncture 
site is possible can aid in making a decision as to whether VAIVT is indicated.

Regarding venous pressure in cases of AVG, statistics regarding instances of low venous pressure in the past or the venous pressure immediately after a previously successful VAIVT should be used as the baseline. Some are of the opinion that cases in which venous pressure elevates $50 \mathrm{mmHg}$ or more past the baseline should be considered cases of significant venous pressure elevation.

Poor maturation immediately after creation of a VA $\mathrm{VA}^{11,27,69,72,88}$ is generally recognized when development remains poor 1 month after VA creation. Occlusion immediately after VA creation is also considered an indication for VAIVT, but in such cases VAIVT should be performed at least two weeks after VA construction.

The descriptions contained herein are in conformance with the contents of the Japanese Society for Dialysis Therapy Guideline, Chapter 5 Managing Trouble Related to Vascular Access (1) Stenosis \& Occlusion GL-1, 2, 3, 5.

CQ2. What are the contraindications for VAIVT?

VAIVT is contraindicated in cases in which the vascular access (AVF, AVG) has become infected, cases in which AVF with old non-thrombotic occlusion in a surface vein at the elbow causes thrombotic occlusion, and cases in which the main outflow route downstream from a site of thrombotic occlusion develops a non-thrombotic occlusion (AVG, AVG).

\section{Recommendation level: $1 \mathrm{~B}$}

\section{Description}

1. Contraindications

In cases of infected $\mathrm{VA}^{3,6,91}$, and particularly cases of thrombotic occlusion of infected AVG, the occluded zone is usually long and the volume of infectious agents is usually high. Therefore, if passage is restored by VAIVT, the infectious agents or other causative agents can spread the infection throughout the body via the bloodstream, which in a worst-case scenario can lead to septicemia. Thus, VAIVT is contraindicated in such cases.

\section{Relative contraindications}

In cases of typical AVF in the forearm, old non-thrombotic occlusions both the cephalic vein route and the basilic vein route is present around the elbow prior to thrombotic occlusion. In cases of typical AVF in the forearm, an old non-thrombotic occlusion is present at some location along the cephalic vein route or the basilica vein route from the forearm near the elbow to the upper arm prior to occlusion. As the deep perforating branches are the only outflow routes just below the elbow, chronic venous hypertension can lead to marked dilation of the veins in the forearm. If a thrombotic occlusion develops under these conditions, the vein often becomes occlude the AVF thrombotic occlusion d throughout its entire length along the forearm and multiple occlusions can occur. Even if the thorombotic occlusion of AVF is restored by VAIVT, it can be difficult to ensure satisfactory blood flow, and even a small amount of residual thrombus can lead to complete interruption of the blood flow in the deep perforating veins, which in turn leads to re-occlusion. Thus, VAIVT is rarely indicated in such cases. ${ }^{\mathrm{J}-2}$

\section{Others}

In cases in which an aneurysm causes markedly poor outflow and decompression of the aneurysm is not possible, VAIVT is not indicated. Moreover, in cases of steal syndrome, if treating the lesion is associated with the risk of further decreasing blood flow to the peripheral finger, VAIVT is not indicated.

In some cases of central vein stenosis/occlusion, a satisfactory collateral route has already been established, and if the only symptom is swelling of the arm, the puncturing performed as part of dialysis will have no effect. Thus, if the patient is not experiencing pain or discomfort, VAIVT is not indicated.

The descriptions contained herein are in conformance with the contents of the Japanese Society for Dialysis Therapy Guideline, Chapter 5 Managing Trouble Related to Vascular Access (1) Stenosis \& Occlusion GL-4 and (2) Aneurysms GL-2, 3, 4.

\section{CQ3. What methods are used to evaluate vascular access?}

Assessment of access failures is in principle based on clinical features (difficulty removing blood, swelling of the access branch, delayed hemostasis, pain in the access branch, etc.) and physiological findings. Evaluation of diagnostic imaging is performed using extracorporeal ultrasound and angiography.

\section{Recommendation level: $1 \mathrm{~A}$}

\section{Description}

Since vascular access utilizes surface blood vessels, most evaluations can be performed using physiological findings and ultrasound. However, because evaluation of stenosis and occlusion can be incomplete when using pathological findings, ultra- 
sound examination is required due to its high degree of objectivity. Ultrasound examination can be used to perform simultaneous functional and morphological evaluations. Angiography is used to assess anterograde and retrograde blood flow. ${ }^{1,2,3,7,10,27}$ Ultrasound is essential to evaluate access and to determine the site and method of VAIVT approach. CT and MRI are only performed in cases of central vein lesions..$^{14,78,79}$

For details regarding the use of ultrasound and angiography, see General Considerations of VAIVT, 4. Diagnostic images required to evaluate VA function.

Physicians who perform VAIVT must have a high degree of skill and experience in the examination of VA dysfunction, in the use of ultrasound images to evaluate VA function, and in appropriate imaging studies.

The descriptions contained herein are in conformance with the contents of the Japanese Society for Dialysis Therapy Guideline, Chapter 4 Routine Management of Vascular Access (3) Surveillance and Monitoring of VA Function GL-6 and Chapter 5 Managing Trouble Related to Vascular Access (3) Venous Hypertension GL-2.

\section{CQ4. What approaches are used to perform VAIVT?}

Although the venous approach is most common, the arterial approach is used in some cases of lesions. AVG is most commonly performed through the graft. In cases of thrombotic occlusion in particular, the sheath is inserted from two facing punctures located in the graft.

\section{Recommendation level: 1B}

\section{Description}

1. Approach

VAIVT is most commonly performed to traverse a guidewire through the site of stenosis or occlusion. The venous approach has a lower risk of arterial dissection and hematoma than the arterial approach. As large-bore sheaths of 6Fr. or larger can be inserted, they are recommended. $6,7,11,12,15,31,34$ When the venous approach is utilized but the guidewire cannot be traversed through the stenosis site and in cases in which performing the procedure on a vein is made difficult by deterioration downstream of the VA, the arterial approach may be required. In addition, in many cases of poor maturation immediately after creation of VA, a stenosis exists in the arteriovenous anastomosis, and this causes insufficient dilation of the vein. In such cases, the arterial approach from the upstream side is useful.

\section{AVG}

In most cases, AVG is performed using an approach through the graft. Stenoses usually develop at the site of the venous anastomosis of the graft. As a result, the thrombus tends to form within the graft. ${ }^{20,25,32}$ In order to ensure satisfactory inflow and outflow in such cases, sheaths are inserted from opposite sides of the graft on the upstream and downstream sides to perform PTA and thrombectomy. In cases in which the sheath insertion sites are limited and lesions are present on the upstream and/or downstream sides, it may be difficult to insert two sheaths. In such cases, it can be possible to use a guidewire to insert one sheath using the inverse method.

The descriptions contained herein are in conformance with the contents of the Japanese Society for Dialysis Therapy Guideline, Chapter 5 Managing Trouble Related to Vascular Access (1) Stenosis and Occlusion GL-2, 3, 4, 5.

\section{CQ5. How are balloon catheters selected?}

Many VA lesions have poor expandability. In such cases, the use of a high pressure-resistant balloon is preferable. For lesions with poor expandability, the use of a cutting balloon may also be considered.

\section{Recommendation level: $\mathbf{1 C}$}

\section{Description}

1. Characteristics of VA stenosis

As VA stenoses are more often lesions with poor expandability than arteriosclerotic lesions, the basic device used in such cases is a high pressure-resistant balloon catheter. ${ }^{51,69,76}$ For lesions expected to have poor expandability, such as in cases of recurrence of previous lesions with poor expandability or lesions located in AVG venous anastomosis sites, the use of ultrahigh pressure-resistant balloon catheters designed to withstand up to $30 \mathrm{~atm}$ or cutting balloon catheters may be considered. ${ }^{1,19,20,29}$ However, since balloon catheter dilation straightens the blood vessel, use on lesions that have pronounced curves is contraindicated.

2. Types and uses of balloon catheters

Types of balloon catheters include non-compliant balloons and semi-compliant balloons. The former expands to a fixed size 
regardless of the pressure applied and, as a result, pressure is applied within the balloon's specified range of pressure resistance until it has reached its predetermined size. If the correct size balloon is selected, there is little danger of damaging the blood vessel due to over-inflation.

For most common types of lesions, either non-compliant or semi-compliant balloons may be utilized.

Since the approached lesions often follow a highly circuitous and complex course and have non-physiologically sharp branch angles at the site of anastomosis, VAIVT needs to be performed using balloon catheters that are highly pressure resistant, that are thin and thus have a low profile, and that are highly trackable, with high ability to traverse such a complex route. .1,76 $^{1,7}$

It is preferable to use semi-compliant balloon catheters on lesions that curve in the juxta-anastomotic site since they cause less straightening of the blood vessel when dilated.

There are general type balloon catheters with internal lumen diameters of 0.035 inches and specialized balloon catheters with internal lumen diameters of below 0.0018 inches. The specialized balloon catheters include both cutting balloon catheters and slip-prevention type balloon catheters. Both types have blades and wires attached in order to make incisions into the hyperplastic intima so as to ensure sufficient dilation., ${ }^{1,23,46,51,73}$

Few RCTs investigate the relation between the various types of catheters and prolonged patency. ${ }^{61,73}$

The descriptions contained herein are in conformance with the contents of the Japanese Society for Dialysis Therapy Guideline, Chapter 5 Managing Trouble Related to Vascular Access (1) Stenosis and Occlusion GL-1, 2, 3, 4, 5.

\section{CQ6. How is the balloon diameter selected?}

Either a balloon diameter equal in size to the venous diameter under compression by blood pressure cuff or $1 \mathrm{~mm}$ oversized is selected for venous lesions with normal blood vessel diameter at the site of the stenosis. In the case of AVF anastomosis and arterial lesions, balloons of the same diameter as the normal blood vessel diameter in the vicinity of the stenosis are selected.

\section{Recommendation level: $1 \mathrm{~B}$}

\section{Description}

In the case of arterial lesions, balloon catheters of nearly the same diameter as the diameter of the normal blood vessel regions at the site of the lesion are selected in order to prevent blood vessel damage such as dissection due to overinflation. In the case of venous lesions, the balloon is dilated until its waist disappears. Since the stenosis may remain due to a elastic recoil phenomenon that commonly occurs after dilation, a balloon catheter size that is slightly larger than the diameter of the normal blood vessel regions at the site of the lesion is often selected. Different vessel diameters on the arterial and venous sides of lesions that involve an AVF arteriovenous anastomosis are sometimes observed. In such cases, the use of balloon diameters same as the vessel diameter on the normal arterial side are prioritized. $\mathrm{Jl}^{\mathrm{J1}, 31,62,63,64,65,66}$

The descriptions contained herein are in conformance with the contents of the Japanese Society for Dialysis Therapy Guideline, Chapter 5 Managing Trouble Related to Vascular Access (1) Stenosis and Occlusion GL-1, 2, 3, 4, 5.

\section{CQ7. How are percutaneous transluminal angioplasty balloons dilated?}

As a general rule, balloons are inflated under their rated burst pressure (RBP) until they lose their waists, which usually takes 30 to 120 seconds. However, in cases of vessel dissection, dilation is performed over longer periods of time at a low pressure.

\section{Recommendation level: $1 \mathrm{C}$}

\section{Description}

There are currently no standards regarding dilation methods. Many VA lesions have poor expandability and therefore higher degrees of dilation than in the case of PAD arterial stenosis are required. Dilation is performed within the rated burst pressure (RBP) but 2 to $4 \mathrm{~atm}$ higher than required to for the waist to disappear. This normally takes 60 seconds. As spasms may occur when dilating an arterial stenosis from the anastomosis site, it is preferable to use low pressure during dilation. There are a variety of expert opinions regarding dilation methods..$^{34,35,36,41,62}$ A study that compared the post-VAIVT patency of a 60 second dilation group and a 180 second dilation group reported no significant difference in long-term patency. ${ }^{55,61}$ In many cases, balloon inflation is continued for 30 to 120 seconds. According to a report by the Japanese Society of Interventional Radiology, 55\% of the survey respondents reported that pressurization at $15 \mathrm{~atm}$ or above was required while $23 \%$ reported that pressurization at 20 atm or more was required..$^{94}$ Regarding balloon inflation duration, most investigators (49\%) reported 60 seconds, followed by 120 seconds (25\%). Regarding the number of inflations, most investigators (27\%) reported two times, followed by three times $(17 \%)$, and one time $(15 \%)$.

In cases in which dissection of the lesion is observed after dilation, long time dilation of at least three minutes at a pressure under the RBP is attempted. ${ }^{94}$

The descriptions contained herein are in conformance with the contents of the Japanese Society for Dialysis Therapy Guide- 
line, Chapter 5 Managing Trouble Related to Vascular Access (1) Stenosis and Occlusion GL-1, 2, 3, 4, 5.

\section{CQ8. What is the endpoint for balloon dilation procedure?}

In general, the goal is to dilate until the balloon waist disappears or the percentage of remnant stenosis is $30 \%$ or below. In cases of AVF, the goal is to dilate until the thrill can no longer be heard or improves.

\section{Recommendation level: $1 \mathrm{C}$}

\section{Description}

The balloon PTA endpoint is to dilate until the waist of the balloon is observed to have disappeared according to fluoroscopy during the procedure. However, there are cases in which dilation at the rated burst pressure (RBP) does not result in the disappearance of the waist, such as when there is a high degree of fibrosis at the stenosis site or when calcification or other structural changes at the stenosis site prevent balloon compliance. There are also cases in which remnant stenosis occurs due to post-dilation elastic recoil..$^{55,61, \mathrm{~J} 1}$

There are both clinical and hemodynamic endpoints. The important point is the appearance of satisfactory thrill. If a satisfactory thrill appears, the residual stenosis is within the allowable range of approximately $30 \%$. The endpoint used by many studies for the degree of residual stenosis as observed using contrast imaging is $30 \%$ or below. ${ }^{44,45,82}$ Even if remnant stenosis is observed, the procedure is completed if a thrill appears or there is improvement.

Even if angiography indicates improvement, arterial spasms, formation of thrombi, and stenosis on the downstream side of the site of dilation are possible if the thrill does not appear. Thus, in such cases the cause must be determined before the procedure is concluded. In cases that present clear dissection, the dissection is compressed using long-term inflation at low pressure ( 4 to 6 atm for 2 to 3 minutes). This prevents the formation of a thrombus in the dissection lumen. ${ }^{90,91,92,93}$

After the procedure is concluded, the activated clotting time (ACT) is measured. When it is 140 seconds or under, protamine sulfate is used to achieve partial neutralization. In order to avoid protamine shock, the protamine sulfate is injected gradually over several minutes. Manual compression after sheath removal is performed at an intensity that is not strong enough to cause bleeding from the sheath insertion wound but just strong enough so that the thrill at the anastomosis site can be detected by palpation. Hemostasis can be achieved with manual compression for 5 to 15 minutes, after which a hemostasis band is used to apply light pressure for 10 minutes. While hemostasis is achieved in the same way even when the brachial artery approach is utilized, particular care is required to avoid applying pressure on the median nerve.

The descriptions contained herein are in conformance with the contents of the Japanese Society for Dialysis Therapy Guideline, Chapter 5 Managing Trouble Related to Vascular Access (1) Stenosis and Occlusion GL-1, 2, 3, 4, 5.

\section{CQ9. How is blood vessel damaged caused by the PTA balloon managed?}

Procedures differ depending upon the degree of blood vessel damage. In cases in which the hematoma does not increase in size and blood flow is not affected, only pain control is required. In present extravasation that affects the blood flow, a balloon catheter is inserted to the extravasation site and the fingers and the balloon are used to apply low level pressure for 4 to 5 minutes. Angiography is used to check for extravasation and blood flow disruption. If repeated balloon dilations and manual compressions do not result in the disappearance of the extravasation as observed on angiography, placement of a stent is considered. Surgical repair is required in cases in which there is complete or partial rupture of the vein.

\section{Recommendation level: $1 \mathrm{C}$}

\section{Description}

Vascular damage is one of the major complications that may arise during balloon dilation. A common cause is when a balloon that is larger than the diameter of the vessel is used. It may occur even when utilizing low-pressure dilation. Patients will complain of pain at the dilation site after balloon deflation following the completion of the balloon dilation procedure. ${ }^{22,32}$

Procedures used to manage damage differ depending upon 1) The juxta-anastomotic site, 2) The vein from the forearm to the upper arm, and 3) The central vein. Regardless of the location of the damage, the guidewire must first not be removed. If a guidewire and the catheter are passed through the ruptured blood vessel later, there is high risk that the guidewire or catheter will be misplaced outside of the blood vessel from the ruptured site. ${ }^{32}$ In addition, neutralization must be immediately performed in cases of heparinization. If possible, activated clotting time (ACT) is measured in order to determine the appropriate dose of protamine sulfate.

1) Juxta-anastomotic site

As a general rule, dilation is performed at low pressure ( $2 \mathrm{~atm}$ ) for between 5 and 10 minutes. ${ }^{22,32,37,45,46,53}$ In cases in which the artery is dissected and occluded due to the damage, stent placement is considered. ${ }^{15,22,32,45,50}$ However, since the radial artery has a small diameter, no stent for peripheral use can be used in this vessel. Therefore, in order to achieve bailout, a coronary 
artery stent must be used. In cases in which a stent cannot be utilized, hemostasis is achieved via balloon dilation, and re-anastomosis must then be performed in a more central region at a later date.

2) Vein from the forearm to the upper arm

In cases of small degree of leakage, dilation is performed between 5 and 10 minutes at 2 atm at the site of the venous rupture and the balloon is simultaneously compressed manually. ${ }^{53}$ If angiography indicates that the leakage has not disappeared after three repetitions of this hemostasis procedure, a self-expandable barestent is installed and the same low-pressure balloon dilation and manual compression procedure as described above is used to stop the leakage from the stent network. If even this additional hemostasis procedure fails to achieve hemostasis, ligation of the shunted blood vessel is considered. Even in cases in which ligation results in the occlusion of the shunt, the likelihood of being able to restore access increases if surgery is performed early.

\section{3) Central vein rupture}

After immediate neutralization using heparin, dilation is performed at the leakage site for 10 minutes at 2 atm. Although manual compression of the leak site is impossible, in many cases manually compression of the vein at the anastomosis site, which acts as an overflow shunt, can decrease the blood flow into the leak site of the central vein and promote hemostasis. ${ }^{22}$ If hemostasis is difficult to achieve using this method, it is still possible that hemostasis can be achieved by the placement of a stent. Although the use of a covered stent is ideal ${ }^{50,57}$, currently in Japan the only appropriate stents are 10 mm diameter biliary stents. If placement of a stent does not achieve hemostasis, the access is ligated after interrupting the blood flow from upstream using a balloon to achieve hemostasis.

The descriptions contained herein are in conformance with the contents of the Japanese Society for Dialysis Therapy Guideline, Chapter 5 Managing Trouble Related to Vascular Access (1) Stenosis and Occlusion GL-1, 2, 3, 4, 5 and (3) Venous Hypertension GL-2.

\section{CQ10. How is thrombotic occlusion treated?}

Thrombolysis therapy using a thrombolysis catheter, urokinase, and t-PA and thrombectomy using a thrombus aspiration catheter are used to treat thrombi. The required sheath size differs depending upon the device used, and the sheath size that can be inserted into the site of interest will limit selection of therapeutic procedures. In the case of AVG, the so-called "lyse-andwait" procedure is performed. In this procedure, urokinase solution is injected into the graft without the use of any devices, and PTA is performed after a designated amount of time has passed. However, since there are cases in which this procedure is contraindicated, care is required when using this procedure.

\section{Recommendation level: $1 \mathrm{C}$}

\section{Description}

1. Treatment methods according to the amount of thrombosis

In cases in which there is a small amount of thrombosis immediately after occlusion, it might be possible to re-establish blood flow with balloon dilation alone. However, if the amount of thrombosis exceeds a certain amount, any thrombectomy must be performed prior to balloon dilatation of the stenotic lesion. Thrombectomy procedures include thrombus aspiration using a vascular sheath with a removable valve ${ }^{15}$, thrombectomy using a thrombus aspiration catheter or large-bore angiographic catheter ${ }^{15,20}$, thrombolysis using a thrombolysis catheter ${ }^{32,69, \mathrm{I}-1, \mathrm{~J}-2}$, or the so-called "lyse-and-wait-" procedure, in which urokinase is injected directly into the graft. ${ }^{5,51,60,70,74,77}$ In cases in which the above procedures cannot be performed, such as when there are small remnant thrombi, a Fogarty catheter with a guidewire lumen can also be used..$^{40}$ In facilities that are able to perform surgical and catheter combined procedures, surgical thrombectomy using a Fogarty catheter and interventional therapy with simultaneous stenosis dilation is another treatment option.

Thrombo-aspiration and thrombectomy require the use of a 6Fr sheath. In cases in which the vessel diameter does not allow the insertion of a 6Fr sheath, the use of thrombolysis that can be performed with a 4Fr sheath is considered. However, since urokinase and t-PA are used for thrombolysis, thrombolysis is contraindicated in some cases.

\section{Contraindications}

1) Patients in whom hemostasis is difficult (intracranial bleeding, expectoration of blood, retroperitoneal bleeding, etc.)

2) Patients who have undergone intracranial or spinal surgery (within the past 2 months)

3) Patients with arterial aneurysm

4) Patients with severe consciousness disorders (because the risk of intracerebral bleeding is high)

5) Patients with or suspected of having cerebral embolism (because hemorrhagic cerebral embolism can occur) 
3. Contraindicated in principle (in principle not performed, but in certain cases in which the procedure is required, it can be performed with caution)

1) Patients with atrial fibrillation (especially mitral valve stenosis), patients with infectious endocarditis, patients with old myocardial infarction, patients with artificial valves

2) Patients that present neurological symptoms of immediate and complete loss of functionality (because of the high risk of cerebral infarction)

4. Performed with care

1) Patients with bleeding: During surgical procedures (including liver and kidney biopsies, etc.), hemorrhagic ocular disease such as diabetic and hemorrhagic retinopathy, gastrointestinal bleeding, urinary tract bleeding, miscarriage/premature birth, immediate postpartum bleeding, excessive bleeding during menstruation, etc.

2) Patients at risk of bleeding: History of gastrointestinal ulcers, diverticulitis, colitis, severe hypertension, vasoactivity, intracranial bleeding, etc.

3) Patients with intractable hypocoagulability: Coagulation factor deficiency, thrombocytopenia, etc.

4) Patients who have been administered medicines with an anticoagulant effect, drugs with a platelet aggregation inhibitory effect, or other thrombolytic agents

In facilities that are able to perform combination therapy involving both surgical procedures and interventional therapies, surgical thrombectomy using a Fogarty catheter and endovascular intervention with simultaneous stenosis dilation is a treatment option.

The descriptions contained herein are in conformance with the contents of the Japanese Society for Dialysis Therapy Guideline, Chapter 5 Managing Trouble Related to Vascular Access (1) Stenosis and Occlusion GL-3, 4, 5.

\section{CQ11. What is the endpoint for thrombectomy?}

In the case of AVF, if a thrill or a strong pulse appears in the site of thrombotic occlusion, and if angiography or ultrasound indicate a marked decrease in the amount of thrombosis, a shift to PTA is made. As in AVF, in the case of AVG as well, if marked reduction in the thrombosis at the thrombotic occlusion site is confirmed, a shift to PTA is made. After balloon PTA, it is essential that a check of the rapid run-off of contrast medium be made.

\section{Recommendation level: $1 \mathrm{C}$}

\section{Description}

As thrombus treatment progresses, the amount of thrombus decreases and communication with the artery is restored. Once this occurs, the pulse appears both in the case of AVF and AVG. At this point, preferably angiography or ultrasound is used to confirm that the residual thrombus has decreased in volume. However, balloon PTA is performed in cases in which the thrombosis remains in spite of the use of a thrombectomy device and thrombolysis urokinase. In cases in which there is a large number of hard residual thrombus at the site of the arteriovenous anastomosis in the case of AVF or the graft-artery anastomosis in the case of AVG, dilation using balloon PTA often results in early post-treatment re-occlusion. Therefore, it is advisable to aspirate the thrombus by Fogarty catheter with the guidewire lumen downstream prior to performing balloon PTA.

The additional treatment of the thrombosis is performed, when the residual thrombosis prevents satisfactory blood flow after balloon dilatation. This involves repeating balloon dilation and thrombosis treatment until satisfactory image findings and physiological findings are confirmed. In rare cases, novel thrombi appear during the course of balloon dilation. In such cases, additional heparin administration and balloon dilation along the entire length of the occlusion is performed in as short a time as possible.

If, after balloon dilation, the thrill can be palpated at the graft near the arteriovenous anastomosis in the case of AVF or the artery anastomosis in the case of AVG, angiography shows satisfactory run-off of contrast medium ${ }^{15}$ or ultrasound shows satisfactory restoration of brachial artery blood flow volume or graft blood flow volume, the procedure may be concluded. If the procedure is concluded without confirming satisfactory pathological or image findings, the probability of early re-occlusion is high.

The descriptions contained herein are in conformance with the contents of the Japanese Society for Dialysis Therapy Guideline, Chapter 5 Managing Trouble Related to Vascular Access (1) Stenosis and Occlusion GL-3, 4, 5. The Guideline has no listings related to stents.

\section{CQ12. What are the indications for stents?}

In cases of elastic recoil, cases of repeated re-stenosis in under three months, and cases of vascular damage in which blood flow does not improves after long time balloon inflation, blood flow dysfunction of the central vein due to anatomical compression is indicated, and in some cases, residual thrombosis or pseudoaneurysm accompanied with the blood flow dysfunction may 
be indicated.

\section{Recommendation level: $1 \mathrm{C}$}

\section{Description}

It is necessary to keep in mind that the appropriate stent placement site is preferably a site above the elbow in a central vein that is not a potential puncture site. Although there is some debate over indications, currently indications on which there is a relative amount of consensus are as follow ${ }^{3,4,12,13,14,15,30,34,64,66,67,83,84}$

1. Cases of elastic recoil in which at least $30 \%$ of the stenosis remains regardless of the fact that full expansion of a balloon of appropriate diameter was possible. This is not to be considered as to mean that a stent is indicated in all cases. In cases in which physiological findings improve, additional treatment is not indicated even if there is residual stenosis.

2. Cases in which re-stenosis occurs in less than three months ${ }^{89}$

If the site of repeated stenosis is in the vicinity of the anastomosis in the forearm, surgical re-establishment of the anastomosis should be considered. There are reports of the prolongation of re-stenosis as a result of stent placement. However, if temporary intra-stent stenosis occurs, normal balloon dilation alone cannot be expected to achieve sufficient expansion.

3. Cases of vascular damage in which hemostasis is difficult

In cases in which hemostasis is difficult to achieve with low-pressure dilation using a balloon catheter and manual compression, stent placement should be considered. Placement of a self-expandable barestent and additional low-pressure balloon dilation with manual compression has a high probability of achieving hemostasis. ${ }^{17}$

4. Damage to the central vein caused by anatomical compression ${ }^{85}$

Compression due to cervico-clavicular junction at the subclavian vein and external compression of the innominate vein by the brachiocephalic artery will make it impossible to achieve sufficient dilation with balloon dilation alone. Stent placement should be considered in such cases.

\section{Other}

In the cases of pseudoaneurysms, aneurysm occlusion is achieved using a covered stent; however, in cases in which the aneurysm is in the vicinity of the anastomosis, aneurysmectomy and re-anastomosis are treatment options.

The Japanese Society for Dialysis Therapy Guideline has no listing related to stents.

\section{CQ13. How are stents selected?}

As a general rule, self-expandable stents are used. However, the use of balloon-expandable stents is also possible in some central veins. Stent diameter is 1 to $2 \mathrm{~mm}$ larger than normal vessel diameter.

\section{Recommendation level: $1 \mathrm{C}$}

\section{Description}

\section{Types of stents}

Stents are divided into self-expandable stents and balloon-expandable stents. While self-expandable stents have a variety of features, all use radial force to dilate the vessel wall. However, in cases of severe fibrosis, i.e., hard lesions, sufficient dilation cannot be achieved. In such cases, the post balloon inflation is necessary in order to attach the stent to vessel wall. As balloon-expandable stents cannot dilate by themselves, a balloon is used to attach the stent to the vessel wall for full-expansion of stent. As a result, they are susceptible to external pressure and cannot re-expand without assistance if they become deformed. In the case of the venous stenosis of extremities, external compression (e.g., trauma) and muscle forces with joints flexion may be applied to the stent, and since blood vessels have different diameters, self-expandable stents are most commonly used. $3,4,9,12,14,15,17,18,20,28,40,42,47,49,50,60,63,66,70,78$ However, in the case of central veins external pressure is not a concern and therefore balloon-expandable stents may be used. ${ }^{1}$

\section{Selection of stent diameter}

There is no clear evidence regarding the issue of stent diameter. However, many studies ${ }^{3,20,30,70}$ report the use of stents that are 1 to $2 \mathrm{~mm}$ larger in diameter, which is the same diameter as normal blood vessels in the vicinity. ${ }^{2}$ The length should be at least as long as the stenosis, but due to the possibility that the edge or the stent ends may cause the development of new stenosis, the stent length should be as close to the stenosis length as possible. ${ }^{2,20,30}$ In some cases, covered stents (stent grafts) are used on pseudoaneurysms due to central vein rupture. ${ }^{1,49}$

The Japanese Society for Dialysis Therapy Guideline has no listing related to stents. 


\section{CQ14. How are stents placed?}

Stents should be placed either directly or indirectly in a way that does not interfere with access creation in the future.

\section{Recommendation level: $1 \mathrm{C}$}

\section{Description}

The vein running from the forearm where the stent is inserted to the upper arm cannot be used to create an arteriovenous anastomosis for access. Thus, in order to preserve a future anastomosis site, stent placement must be carefully considered. As a general rule, it must be kept in mind that the stent placement site should be in a central vein above the elbow in a site that is not used as a puncture site.

When placing a stent in the subclavian vein, in order to ensure a future access route, it should not overlap the opening of the internal jugular vein in which patency has been achieved..$^{30,34,38}$ Since stenosis might be caused in the main trunk of the brachiocephalic vein on the opposing side when placing a stent in the brachiocephalic vein on one side, the stent should be placed so that it does not protrude into the superior vena cava. In cases of posterior dilation in which it is necessary to ensure that the stent adheres to the blood vessel wall, a balloon catheter should be used prior to stent placement to perform posterior dilation.

Stents can be either balloon-expandable type or self-expandable type. A self-expandable stent is commonly used at sites of flexion and access vessels that have different blood vessel diameters. ${ }^{3,4,9,12,14,15,17,18,20,28,40,42,47,49,50,60,63,66,70,78}$ In the approach, as a 6Fr or larger sheath is required, a blood vessel that can accept that size sheath is also required. The venous approach is most common. At the forearm level, the retrograde approach is used, and in the case of a central vein, the anterograde approach is used. Selection of stent diameter is determined by the diameter of normal blood vessels in the vicinity.

The Japanese Society for Dialysis Therapy Guideline has no listing related to stents.

\section{CQ15. What complications are associated with stent installation?}

The same treatment as used in cases of common thrombotic occlusion is used in cases of intra-stent thrombotic occlusion. Recovery of migrated stents is attempted using a snare catheter or other type of catheters designed to remove foreign bodies. Additional stent placement is performed as necessary in cases of stent shrinkage, damage, or migration.

\section{Recommendation level: $1 \mathrm{C}$}

\section{Description}

Although the treatment for intra-stent thrombotic occlusion is the same treatment as is used for common thrombotic occlusion, intra-stent thrombi in a central vein may flow into the lungs without any decrease in size and are liable to cause severe pulmonary infarction. Thus, extreme care is required when handling such thrombi.

When deploying a stent in a central vein, it is advisable to prevent migration in the heart if possible by performing the procedure after a guidewire pull-through. Even if the stent is migrated in the vena cava, if this occurs after pull-through, it will not enter the ventricle via the atrium. In cases in which the stent is migrated in the vena cava or heart, a snare catheter or other catheter designed to remove foreign bodies is used to safely transfer the migrated stent into a sheath and then remove the sheath by cutting down the vessel. ${ }^{20}$ If possible, movement to safe location such as between the brachiocephalic vein and the subclavian vein or the iliac vein is required. If movement to a safe location is possible, stable placement at that site is attempted. If stable placement in a safe location is difficult, fixation by placement of an additional stent may be performed. Additional stent placement $^{49}$ is considered in cases of stent shortening, damage, or localized migration ${ }^{70}$ if these conditions cause a lesion to develop or symptoms to appear.

The Japanese Society for Dialysis Therapy Guideline has no listing related to stents.

\section{References related to General Considerations}

1) Technical Terms of Dialysis Therapy. J. Jpn. Soc. Dial. Ther. 40 (12): 957-1023, 2007

2) Guidelines of creation and repair for vascular access of dialysis therapy. J. Jpn. Soc. Dial. Ther. 44 (9): 855-938, 2011.

3) Ohhira Seiji, et al.: Vascular Access: The creation, maintainance, and repair, Chugaiigakusha, 2007. (in Japanese).

4) Dheeraj K. Rajan, et al.: Essentials of Percutaneous dialysis Interventios, Springer Science, 2011.

5) Rodrigues L.T, Renaud C.J.: Diagnostic and Interventional Radiology of Arteriovenous Accesses for Hemodialysis, Springer-Verlag France, 2013.

6) Agishi Tetsuzou, et al.: Vascular Access Intervention, Shuujunsha, Shuujunsha, 2013. (in Japanese).

7) Annual dialysis data report 2015, JSDT Registry, J. Jpn. Soc. Dial. Ther. 50 (1): 1-62, 2017.

8) Tsushima Junichi, Gotoh Yasuo, Matsuura Katsuhiko, et al.: A Multicenter Prospective Survey on Image-guided Ebdovascular Management for Non-functioning Hemodialysis Shunt. Jpn J Intervent Radiol, 24: 368-383, 2009. (in Japanese) 
9) Haruguchi Hiroaki, et al.: Vascular Access Ultrasound, Ishiyakuhuppann, 2011. (in Japanese).

\section{References related to the CQs}

1. Kariya S, Tanigawa N, Kojima H, et al.: Percutaneous transluminal cutting-balloon angioplasty for hemodialysis access stenoses resistant to conventional balloon angioplasty. Acta Radiol 2006; 47: 1017-21.

2. Yevzlin AS, Maya ID, Asif A.: Endovascular stents for dialysis access: under what circumstances do the data support their use? Adv Chronic Kidney Dis 2009; 16: 352-9.

3. Vesely TM.: Endovascular intervention for the failing vascular access. Adv Ren Replace Ther 2002; 9: 99-108.

4. Ozyer U, Harman A, Yildirim E, Aytekin C, Karakayali F, Boyvat F.: Long-term results of angioplasty and stent placement for treatment of central venous obstruction in 126 hemodialysis patients: a 10-year single-center experience. AJR Am J Roentgenol 2009; 193 : $1672-9$.

5. Peirce RM, Funaki B, Van Ha TG, Lorenz JM.: Percutaneous declotting of virgin femoral hemodialysis grafts. AJR Am J Roentgenol 2005; 185: 1615-9.

6. Wang HJ, Yang YF.: Percutaneous treatment of dysfunctional Brescia-Cimino fistulae through a radial arterial approach. Am J Kidney Dis 2006; 48: 652-8.

7. Bakken AM, Galaria, II, Agerstrand C, et al.: Percutaneous therapy to maintain dialysis access successfully prolongs functional duration after primary failure. Ann Vasc Surg 2007; 21: 474-80.

8. Bhat R, McBride K, Chakraverty S, Vikram R, Severn A.: Primary cutting balloon angioplasty for treatment of venous stenoses in native hemodialysis fistulas: long-term results from three centers. Cardiovasc Intervent Radiol 2007; 30: 1166-70; discussion 71-2.

9. Denison GL, Funaki B.: Percutaneous mural fenestration and angioplasty for the treatment of a refractory hemodialysis-related venous stenosis. Cardiovasc Intervent Radiol 2006; 29: 1163-4.

10. Fujiwara H, Narimatsu Y, Hashimoto S, et al.: Long-term results of percutaneous transluminal angioplasty for hemodialysis shunt insufficiency. Cardiovasc Intervent Radiol 2000; 23: 83.

11. Hong HP, Kim SK.: Usefulness of percutaneous intervention with transarterial approach in the salvage of nonmaturing native fistulas status-post transvenous approach failure: transarterial approach in the salvage of nonmaturing native fistulas. Cardiovasc Intervent Radiol 2009; 32 : 1252-6.

12. Kariya S, Tanigawa N, Kojima H, et al.: Peripheral stent placement in hemodialysis grafts. Cardiovasc Intervent Radiol 2009 ; 32 : $960-6$.

13. Kim YC, Won JY, Choi SY, et al.: Percutaneous treatment of central venous stenosis in hemodialysis patients: long-term outcomes. Cardiovasc Intervent Radiol 2009; 32: 271-8.

14. Maskova J, Komarkova J, Kivanek J, Danes J, Slavikova M.: Endovascular treatment of central vein stenoses and/or occlusions in hemodialysis patients. Cardiovasc Intervent Radiol 2003; 26: 27-30.

15. Miyayama S, Matsui O, Taki K, et al.: Occluded Brescia-cimino hemodialysis fistulas: endovascular treatment with both brachial arterial and venous access using the pull-through technique. Cardiovasc Intervent Radiol 2005; 28: 806-12.

16. Peregrin JH, Rocek M.: Results of a peripheral cutting balloon prospective multicenter European registry in hemodialysis vascular access. Cardiovasc Intervent Radiol 2007; 30: 212-5.

17. Rajan DK, Clark TW. P: Patency of Wallstents placed at the venous anastomosis of dialysis grafts for salvage of angioplasty-induced rupture. Cardiovasc Intervent Radiol 2003; 26: 242-5.

18. Rajan DK, Saluja JS.: Use of nitinol stents following recanalization of central venous occlusions in hemodialysis patients. Cardiovasc Intervent Radiol 2007; 30: 662-7.

19. Shin SW, Do YS, Choo SW, Lieu WC, Choo IW.: Salvage of immature arteriovenous fistulas with percutaneous transluminal angioplasty. Cardiovasc Intervent Radiol 2005; 28: 434-8.

20. Turmel-Rodrigues L, Pengloan J, Bourquelot P.: Interventional radiology in hemodialysis fistulae and grafts: a multidisciplinary approach. Cardiovasc Intervent Radiol 2002; 25: 3-16.

21. Verstandig AG, Bloom AI, Sasson T, Haviv YS, Rubinger D.: Shortening and migration of Wallstents after stenting of central venous stenoses in hemodialysis patients. Cardiovasc Intervent Radiol 2003; 26: 58-64.

22. Bittl JA.: Venous rupture during percutaneous treatment of hemodialysis fistulas and grafts. Catheter Cardiovasc Interv 2009; 74 : $1097-101$.

23. Bittl JA, Feldman RL.: Cutting balloon angioplasty for undilatable venous stenoses causing dialysis graft failure. Catheter Cardiovasc Interv 2003; 58: 524-6.

24. Bittl JA, von Mering GO, Feldman RL.: Adaptive remodeling of hypoplastic hemodialysis fistulas salvaged with angioplasty. Catheter Cardiovasc Interv 2009; 73: 974-8.

25. Lai CC, Kang PL, Tsai HL, Mar GY, Liu CP.: Percutaneous management of acutely thrombosed hemodialysis grafts: the double balloon occlusion technique. Catheter Cardiovasc Interv 2009; 73: 117-22.

26. Chan MR, Bedi S, Sanchez RJ, et al.: Stent placement versus angioplasty improves patency of arteriovenous grafts and blood flow of arteriovenous fistulae. Clin J Am Soc Nephrol 2008; 3: 699-705.

27. Nassar GM, Nguyen B, Rhee E, Achkar K.: Endovascular treatment of the "failing to mature” arteriovenous fistula. Clin J Am Soc Nephrol 2006; 1: $275-80$.

28. Yevzlin A, Asif A.: Stent placement in hemodialysis access: historical lessons, the state of the art and future directions. Clin J Am Soc Nephrol 2009; 4: 996-1008.

29. Ono T, Goto H, Morishita K, Kondo H, Hirakata H, Iida M.: Immediate hemodialysis after percutaneous transvenous angioplasty increases patency rates of arterio-venous fistula. Clin Nephrol 2004; 62: 301-5.

30. Aytekin C, Boyvat F, Yagmurdur MC, Moray G, Haberal M.: Endovascular stent placement in the treatment of upper extremity central venous obstruction in hemodialysis patients. Eur J Radiol 2004; 49: 81-5. 
31. Maeda K, Furukawa A, Yamasaki M, Murata K.: Percutaneous transluminal angioplasty for Brescia-Cimino hemodialysis fistula dysfunction: technical success rate, patency rate and factors that influence the results. Eur J Radiol 2005; 54: 426-30.

32. Sofocleous CT, Schur I, Koh E, et al.: Percutaneous treatment of complications occurring during hemodialysis graft recanalization. Eur J Radiol 2003; 47: 237-46.

33. Kakkos SK, Haddad GK, Haddad JA, Scully MM.: Secondary patency of thrombosed prosthetic vascular access grafts with aggressive surveillance, monitoring and endovascular management. Eur J Vasc Endovasc Surg 2008; 36: 356-65.

34. Karakayali F, Basaran O, Ekici Y, et al.: Effect of secondary interventions on patency of vascular access sites for hemodialysis. Eur J Vasc Endovasc Surg 2006; 32: 701-9.

35. Haage P, Krings T, Schmitz-Rode T.: Nontraumatic vascular emergencies: imaging and intervention in acute venous occlusion. Eur Radiol 2002; 12: $2627-43$.

36. Vorwerk D.: Non-traumatic vascular emergencies: management of occluded hemodialysis shunts and venous access. Eur Radiol 2002; 12 : 264450 .

37. Miquelin DG, Reis LF, da Silva AA, de Godoy JM.: Percutaneous transluminal angioplasty in the treatment of stenosis of arteriovenous fistulae for hemodialysis. Int Arch Med 2008; 1: 16.

38. Chen CY, Liang HL, Pan HB, et al.: Metallic stenting for treatment of central venous obstruction in hemodialysis patients. J Chin Med Assoc 2003; 66: 166-72.

39. Anain P, Shenoy S, O’Brien-Irr M, Harris LM, Dryjski M.: Balloon angioplasty for arteriovenous graft stenosis. J Endovasc Ther 2001; 8: 16772.

40. Kakkos SK, Haddad GK, Haddad J, Scully MM.: Percutaneous rheolytic thrombectomy for thrombosed autogenous fistulae and prosthetic arteriovenous grafts: outcome after aggressive surveillance and endovascular management. J Endovasc Ther 2008; 15: 91-102.

41. Tynan-Cuisiner G, Berman SS.: Advances in endovascular techniques to treat failing and failed hemodialysis access. J Endovasc Ther $2004 ; 11$ Suppl 2: II134-9.

42. Tan CW, Yount RD, Quintal RE.: Percutaneous recanalization of thrombosed dialysis shunts. J La State Med Soc 2000; 152 : 559-62.

43. Clark TW.: Nitinol stents in hemodialysis access. J Vasc Interv Radiol 2004; 15: 1037-40.

44. Cohen A, Korzets A, Neyman H, et al.: Endovascular interventions of juxtaanastomotic stenoses and thromboses of hemodialysis arteriovenous fistulas. J Vasc Interv Radiol 2009; 20: 66-70.

45. Falk A.: Maintenance and salvage of arteriovenous fistulas. J Vasc Interv Radiol 2006; 17: 807-13.

46. Guiu B, Loffroy R, Ben Salem D, et al.: Angioplasty of long venous stenoses in hemodialysis access: at last an indication for cutting balloon? J Vasc Interv Radiol 2007; 18: 994-1000.

47. Gupta M, Rajan DK, Tan KT, Sniderman KW, Simons ME.: Use of expanded polytetrafluoroethylene-covered nitinol stents for the salvage of dysfunctional autogenous hemodialysis fistulas. J Vasc Interv Radiol 2008; 19: 950-4.

48. Manninen HI, Kaukanen E, Makinen K, Karhapaa P.: Endovascular salvage of nonmaturing autogenous hemodialysis fistulas: comparison with endovascular therapy of failing mature fistulas. J Vasc Interv Radiol 2008; 19: 870-6.

49. Nael K, Kee ST, Solomon H, Katz SG.: Endovascular management of central thoracic veno-occlusive diseases in hemodialysis patients: a single institutional experience in 69 consecutive patients. J Vasc Interv Radiol 2009; 14: 46-51.

50. Rajan DK, Clark TW, Patel NK, Stavropoulos SW, Simons ME.: Prevalence and treatment of cephalic arch stenosis in dysfunctional autogenous hemodialysis fistulas. J Vasc Interv Radiol 2003; 14: 567-73.

51. Rajan DK, Platzker T, Lok CE, et al.: Ultrahigh-pressure versus high-pressure angioplasty for treatment of venous anastomotic stenosis in hemodialysis grafts: is there a difference in patency? J Vasc Interv Radiol 2007; 18: 709-14.

52. Rocek M, Peregrin JH, Lasovickova J, Krajickova D, Slaviokova M.: Mechanical thrombolysis of thrombosed hemodialysis native fistulas with use of the Arrow-Trerotola percutaneous thrombolytic device: our preliminary experience. J Vasc Interv Radiol 2000; 11: 1153-8.

53. Singer-Jordan J, Papura S.: Cutting balloon angioplasty for primary treatment of hemodialysis fistula venous stenoses: preliminary results. J Vasc Interv Radiol 2005; 16: 25-9.

54. Song HH, Kim KT, Chung SK, Kim YO, Yoon SA.: Cutting balloon angioplasty for resistant venous stenoses of Brescia-Cimino fistulas. J Vase Interv Radiol 2004; 15: 1463-7.

55. Trerotola SO, Kwak A, Clark TW, et al.: Prospective study of balloon inflation pressures and other technical aspects of hemodialysis access angioplasty. J Vasc Interv Radiol 2005; 16: 1613-8.

56. Turmel-Rodrigues LA.: Hemodialysis access declotting: a native fistula is not a prosthetic graft. J Vasc Interv Radiol 2000; 11: 135-7.

57. Vesely TM, Pilgram TK.: Angioplasty balloon inflation pressures during treatment of hemodialysis graft-related stenoses. J Vasc Interv Radiol 2006; 17: 623-8.

58. Vesely TM, Siegel JB.: Use of the peripheral cutting balloon to treat hemodialysis-related stenoses. J Vasc Interv Radiol 2005; 16: 1593-603.

59. Vogel PM, Parise C. SMART stent for salvage of hemodialysis access grafts.: J Vasc Interv Radiol 2004; 15: 1051-60.

60. Vogel PM, Parise C.: Comparison of SMART stent placement for arteriovenous graft salvage versus successful graft PTA. J Vasc Interv Radiol 2005; 16: 1619-26.

61. Wu CC, Lin MC, Pu SY, Tsai KC, Wen SC.: Comparison of cutting balloon versus high-pressure balloon angioplasty for resistant venous stenoses of native hemodialysis fistulas. J Vasc Interv Radiol 2008; 19: 877-83.

62. Ascher E, Hingorani A, Marks N.: Duplex-guided balloon angioplasty of failing or nonmaturing arterio-venous fistulae for hemodialysis: a new office-based procedure. J Vasc Surg 2009; 50: 594-9.

63. Bakken AM, Protack CD, Saad WE, Lee DE, Waldman DL, Davies MG.: Long-term outcomes of primary angioplasty and primary stenting of central venous stenosis in hemodialysis patients. J Vasc Surg 2007; 45: 776-83.

64. Duijm LE, van der Rijt RH, Cuypers PW, et al.: Outpatient treatment of arterial inflow stenoses of dysfunctional hemodialysis access fistulas by 
retrograde venous access puncture and catheterization. J Vasc Surg 2008; 47: 591-8.

65. Kolakowski S, Jr., Dougherty MJ, Calligaro KD.: Salvaging prosthetic dialysis fistulas with stents: forearm versus upper arm grafts. J Vasc Surg 2003; 38: 719-23.

66. Shemesh D, Goldin I, Zaghal I, Berlowitz D, Raveh D, Olsha O.: Angioplasty with stent graft versus bare stent for recurrent cephalic arch stenosis in autogenous arteriovenous access for hemodialysis: a prospective randomized clinical trial. J Vasc Surg 2008; 48: 1524-31, 31 e1-2.

67. Sprouse LR, 2nd, Lesar CJ, Meier GH, 3rd, et al.: Percutaneous treatment of symptomatic central venous stenosis [corrected]. J Vasc Surg 2004; 39: $578-82$.

68. Turmel-Rodrigues LA.: Regarding "Angioplasty with stent graft versus bare stent for recurrent cephalic arch stenosis in autogenous arteriovenous access for hemodialysis: a prospective randomized clinical trial”. J Vasc Surg 2009; 50: 472; author reply-4.

69. White JJ, Paulson WD, Schwab SJ.: Treatment of hemodialysis AV graft stenosis: stents resurgent. Kidney Int 2005; 67: 772-3.

70. Liang HL, Pan HB, Lin YH, et al.: Metallic stent placement in hemodialysis graft patients after insufficient balloon dilation. Korean J Radiol 2006; 7: 118-24.

71. Kariya S, Tanigawa N, Kojima H, et al.: Residual stenosis after conventional balloon angioplasty for hemodialysis shunt failure: treatment with metallic stent placement and post-balloon dilatation. Radiat Med 2005; 23: 51-5.

72. Clark TW, Cohen RA, Kwak A, et al.: Salvage of nonmaturing native fistulas by using angioplasty. Radiology 2007; $242: 286-92$.

73. Kariya S, Tanigawa N, Kojima H, et al.: Primary patency with cutting and conventional balloon angioplasty for different types of hemodialysis access stenosis. Radiology 2007; 243: 578-87.

74. Liang HL, Pan HB, Chung HM, et al.: Restoration of thrombosed Brescia-Cimino dialysis fistulas by using percutaneous transluminal angioplasty. Radiology 2002; 223: 339-44.

75. Manninen HI, Kaukanen ET, Ikaheimo R, et al.: Brachial arterial access: endovascular treatment of failing Brescia-Cimino hemodialysis fistulas--initial success and long-term results. Radiology 2001; 218: 711-8.

76. Trerotola SO, Stavropoulos SW, Shlansky-Goldberg R, Tuite CM, Kobrin S, Rudnick MR.: Hemodialysis-related venous stenosis: treatment with ultrahigh-pressure angioplasty balloons. Radiology 2004; 231: 259-62.

77. Cynamon J, Pierpont CE.: Thrombolysis for the treatment of thrombosed hemodialysis access grafts. Rev Cardiovasc Med 2002; 3 Suppl 2: S84-91.

78. Kwok PC.: Endovascular Treatment for Central Venous Stenosis due to Central Vein Catheterization for Hemodialysis. Saudi J Kidney Dis Transpl 2004; 15: 338-45.

79. Asif A, Salman L, Carrillo RG, et al.: Patency rates for angioplasty in the treatment of pacemaker-induced central venous stenosis in hemodialysis patients: results of a multi-center study. Semin Dial 2009; 22: 671-6.

80. Beathard GA.: Management of complications of endovascular dialysis access procedures. Semin Dial 2003; 16: 309-13.

81. Beathard GA.: Successful treatment of the chronically thrombosed dialysis access graft: resuscitation of dead grafts. Semin Dial 2006; 19: 41720.

82. Clark TW, Rajan DK.: Treating intractable venous stenosis: present and future therapy. Semin Dial 2004; 17: 4-8.

83. Jain G, Maya ID, Allon M.: Outcomes of percutaneous mechanical thrombectomy of arteriovenous fistulas in hemodialysis patients. Semin Dial 2008; 21: 581-3.

84. Maya ID, Saddekni S, Allon M.: Treatment of refractory central vein stenosis in hemodialysis patients with stents. Semin Dial 2007; $20: 78-82$.

85. Packer J.: An alternative approach to a totally occluded subclavian vein in a malfunctioning arteriovenous fistula. Semin Dial 2007; 20 : $365-9$.

86. Vesely TM, Amin MZ, Pilgram T.: Use of stents and stent grafts to salvage angioplasty failures in patients with hemodialysis grafts. Semin Dial 2008; 21: 100-4.

87. Wusirika R, Leavitt M, Boyer C, et al.: Hemodialysis graft with blind loop inflow segment treated with stent placement. Semin Dial 2008; 21: 455-6.

88. Beathard GA.: Angioplasty for arteriovenous grafts and fistulae. Semin Nephrol 2002; 22: 202-10.

89. Vesely TM.: Role of stents and stent grafts in management of hemodialysis access complications. Semin Vasc Surg 2007; $20: 175-83$.

90. Nassar GM. Endovascular management of the "failing to mature" arteriovenous fistula. Tech Vasc Interv Radiol 2008; 11: 175-80.

91. Mansilla AV, Toombs BD, Vaughn WK, Zeledon JI.: Patency and life-spans of failing hemodialysis grafts in patients undergoing repeated percutaneous de-clotting. Tex Heart Inst J 2001; 28: 249-53.

92. Naoum JJ, Irwin C, Hunter GC.: The use of covered nitinol stents to salvage dialysis grafts after multiple failures. Vasc Endovascular Surg 2006; 40: $275-9$.

93. Surowiec SM, Fegley AJ, Tanski WJ, et al.: Endovascular management of central venous stenoses in the hemodialysis patient: results of percutaneous therapy. Vasc Endovascular Surg 2004; 38: 349-54.

94. Tsushima Junichi, Gotoh Yasuo, Matsuura Katsuhiko, et al.: A Multicenter Prospective Survey on Image-guided Ebdovascular Management for Non-functioning Hemodialysis Shunt. Jpn J Intervent Radiol, 24: 368-383, 2009. 